\title{
Strain-Tunable Electronic and Optical Properties of Monolayer Germanium Monosulfide: Ab-Initio Study
}

\author{
P.T.T. LE, ${ }^{1,2,16}$ CHUONG V. NGUYEN, ${ }^{3}$ DOAN V. THUAN, $, 4,5,17$ \\ TUAN V. VU, ${ }^{6,7}$ V.V. ILYASOV ${ }^{8}$ N.A. POKLONSKI, ${ }^{9}$ HUYNH V. PHUC, ${ }^{10}$ \\ I.V. ERSHOV, ${ }^{8}$ G.A. GEGUZINA, ${ }^{11}$ NGUYEN V. HIEU, ${ }^{12}$ BUI D. HOI, ${ }^{13}$ \\ NGO X. CUONG, ${ }^{14}$ and NGUYEN N. HIEU (D)
}

\begin{abstract}
1.-Laboratory of Magnetism and Magnetic Materials, Advanced Institute of Materials Science, Ton Duc Thang University, Ho Chi Minh City, Viet Nam. 2.-Faculty of Applied Sciences, Ton Duc Thang University, Ho Chi Minh City, Viet Nam. 3.-Department of Materials Science and Engineering, Le Quy Don Technical University, Ha Noi, Viet Nam. 4.-NTT Hi-Tech Institute, Nguyen Tat Thanh University, Ho Chi Minh City, Viet Nam. 5.-Center of Excellence for Green Energy and Environmental Nanomaterials (CE@GrEEN), Nguyen Tat Thanh University, Ho Chi Minh City, Viet Nam. 6.-Division of Computational Physics, Institute for Computational Science, Ton Duc Thang University, Ho Chi Minh City, Viet Nam. 7.-Faculty of Electrical and Electronics Engineering, Ton Duc Thang University, Ho Chi Minh City, Viet Nam. 8.-Department of Physics, Don State Technical University, Rostov on Don, Russia 344000. 9.-Department of Physics, Belarusian State University, Minsk, Belarus. 10.-Division of Theoretical Physics, Dong Thap University, Dong Thap, Viet Nam. 11.-Institute of Physics, Southern Federal University, Rostov on Don, Russia. 12.-Department of Physics, University of Education, The University of Da Nang, Da Nang, Viet Nam. 13.-Department of Physics, University of Education, Hue University, Hue, Viet Nam. 14.-Department of Electrical Engineering, Quang Tri Branch, Hue University, Quang Tri, Viet Nam. 15.-Institute of Research and Development, Duy Tan University, Da Nang 59000, Viet Nam. 16. - -mail: lethithuphuong@tdtu.edu.vn. 17.—e-mail: doanthuanms@gmail.com. 18.—e-mail: hieunn@duytan.edu.vn
\end{abstract}

In the present work, we consider systematically the electronic and optical properties of two-dimensional monolayer germanium monosulfide (GeS) under uniaxial strains along armchair (AC-strain) and zigzag (ZZ-strain) directions. Our calculations show that, at the equilibrium state, the monolayer GeS is a semiconductor with an indirect band gap of $1.82 \mathrm{eV}$. While monolayer GeS is still an indirect band gap semiconductor under ZZ-strain, an indirect-direct energy gap transition can be found in the monolayer GeS when the AC-strain is applied. The optical spectra of the monolayer GeS have strong anisotropy in the investigated energy range from $0 \mathrm{eV}$ to $8 \mathrm{eV}$. Based on optical properties, we believe that the monolayer $\mathrm{GeS}$ is a potential candidate for applications in energy conversion and optoelectronic technologies.

Key words: Monolayer GeS, band gap, optical properties, strain engineering, first-principles calculations

\section{INTRODUCTION}

Recently, the layered monochalcogenides have attracted much attention because of their extraordinary physical properties and prospective applications in spintronic and optoelectronic devices. ${ }^{1-6}$

(Received July 19, 2018; accepted January 18, 2019;

published online February 12, 2019)
Especially, two-dimensional (2D) hexagonal structures of binary IV-VI compounds, such as $\mathbf{M X}(\mathbf{M}=$ $\mathrm{Ge}, \mathrm{Sn}, \mathrm{X}=\mathrm{S}$, Se, Te) monolayers, have seriously been studied because they are a good candidate for applications in energy conversion technologies. ${ }^{7,8}$ Besides, in contrast to the 2D graphene, a semiconductor with zero band gap, ${ }^{9}$ the MX monolayers are semiconductors with nature band gap and their band gap depends strongly on the chalcogenide atom. ${ }^{7}$ 
Compared to the other above-mentioned MX monolayers, monolayer germanium monosulfide $(\mathrm{GeS})$ has the largest band gap, ${ }^{2}$ and the electron mobility of the monolayer GeS is very high, $3680 \mathrm{~cm}^{2} / \mathrm{Vs}^{10}$ Also, the monolayer GeS has high absorption efficiency in the visible light region, which has been widely used in solar cell technology. ${ }^{7}$ The physical properties of bulk GeS have been studied for a long time. ${ }^{11,12}$ Recently, single crystalline GeS nanosheets have been successfully synthesized using different methods. ${ }^{13-15}$ Singh and Hennig predicated theoretically that the GeS monolayer is dynamically stable and can be mechanically exfoliated from bulk. ${ }^{12}$ Also, using first-principles calculations, Chowdhury and coworkers indicated that the monolayer GeS is also stable in an aqueous environment. ${ }^{16}$ By comparing DFT calculated results for formation energy of the monolayer GeS with that of other successfully synthesized monolayers, they expected that the monolayer GeS can be synthesized by experiments. Further, based on theoretical simulations, Wang and Qian believed that the monolayer GeS is thermodynamically stable at room temperature and beyond. ${ }^{17}$ Especially, using first-principles molecular dynamics simulations, $\mathrm{Li}$ and co-worker showed that the geometry of the monolayer GeS can be well kept at very high temperatures, up to $800 \mathrm{~K}^{10}$ This is strong evidence that the monolayer GeS has good thermodynamic stability. Recently, several theoretical works focused on the structural, electronic, and other physical properties of the monolayer GeS. ${ }^{2,7,18}$ Using density functional theory (DFT), Huang and co-workers showed that, similar to the modulation of the band gap, the charge transfer from the metal atom $\mathbf{M}$ to the chalcogenide atom $\mathrm{X}$ in $\mathrm{MX}$ monolayers will decrease with the change of the chalcogenide atom from $\mathrm{S}$ to $\mathrm{Te}^{7}$ Also, earlier DFT calculations indicated that the effect of spin-orbit interaction on the electronic structure of the GeS is negligible. ${ }^{19}$ At the equilibrium state, the monolayer $\mathrm{GeS}$ is an indirect band gap semiconductor with an energy gap of $1.73 \mathrm{eV}$ by DFT within the Perdew, Burke, and Ernzerhof (PBE) functional. ${ }^{7}$ However, the use of functionals greatly influences the outcome of calculations for the energy band gap. ${ }^{2,7}$ Technically, the band gap of the GeS may be changed by engineering strain or external electric field ${ }^{20}$ and the semiconductor-metal phase transition has been observed in the monolayer $\mathrm{GeS}$ at large biaxial uniform strain ${ }^{7}$ or under an external electric field. ${ }^{20}$ Strain engineering is one of the most common methods for manipulating properties of materials, including energy gap, carrier mobility, etc. In the previous studies, they have found that both biaxial and uniaxial strain can be used to control these properties of the monolayer GeS. ${ }^{7,20,21}$ Our recent calculations demonstrated that the band gap of the monolayer $\mathrm{GeS}$ is sensitive to biaxial strain and the semiconductor-metal phase transition has been found at large compression biaxial strain $\varepsilon_{b}{ }^{22}$ The GeS has an orthorhombic structure which belongs to the Pnma 62 space group. ${ }^{19}$ With this structure, the optical properties of the GeS have very high anisotropy. ${ }^{7,23}$ Therefore, the effect of uniaxial strain on the optical properties of GeS is expected to bring many interesting things. However, studies on the monolayer GeS are still lacking and many of its physical properties remain unclear. In this work, we systematically consider the electronic and optical properties of monolayer GeS under the uniaxial strains along individually both the armchair (ACstrain) and zigzag (ZZ-strain) directions using DFT calculations. We focus on the band structure and band gap modulation in the monolayer GeS by uniaxial strains from $-15 \%$ to $15 \%$. We also investigate the basic optical properties of uniaxial strained monolayer $\mathrm{GeS}$ to have further insight into applications in optoelectronic technology.

\section{THEORETICAL MODEL AND METHOD}

In the present work, all our calculations for structural and electronic properties of the monolayer GeS are calculated using the simulation Quantum Espresso package ${ }^{24}$ with the generalized gradient approximations (GGA) of the PerdewBurke- Ernzerhof (PBE) functional. ${ }^{25,26}$ The atomic structure of monolayer GeS is fully relaxed until the remaining force acting on each atom is less than $10^{-6} \mathrm{eV} / \mathrm{A}$. The electronic wave-functions are expanded as plane-wave basis sets with a kinetic energy cut-off of $500 \mathrm{eV}$. For the geometric optimization and calculations of the electronic properties of monolayer $\mathrm{GeS}$, the integration of the Brillouin zone is carried out by a $15 \times 15 \times 1 k$ mesh according to the Monkhorst-Pack grid. The present approach is successfully used for calculations of the similar systems in our previous works. ${ }^{27-30}$

To consider the optical properties of the monolayer GeS, we first estimate the dielectric function $\varepsilon(\omega)=\varepsilon_{1}(\omega)+i \varepsilon_{2}(\omega)$ and then investigate the dependence of the absorption coefficient of monolayer $\mathrm{GeS}$ on the uniaxial strain.

The imaginary part $\varepsilon_{2}(\omega)$ of dielectric function can be expressed as the following ${ }^{6,31}$ :

$$
\begin{aligned}
\varepsilon_{2}^{i j}(\omega)= & \frac{4 \pi^{2} e^{2}}{V m^{2} \varpi^{2}} \sum_{n n^{\prime} \sigma}\left\langle k n \sigma\left|p_{i}\right| k n^{\prime} \sigma\right\rangle\left\langle k n^{\prime} \sigma\left|p_{j}\right| k n \sigma\right\rangle, \\
& \times f_{k n}\left(1-f_{k n^{\prime}}\right) \delta\left(E_{k n^{\prime}}-E_{k n}-\hbar \omega\right),
\end{aligned}
$$

where $\omega$ is the angular frequency of electromagnetic irradiation, $e$ and $m$ are, respectively, the charge and mass of the electron, $V$ is the unit-cell volume, $p$ is the momentum operator, $|k n p\rangle$ is the wave function of a crystal with the wave vector $\vec{k}, f_{k n}$ is the Fermi distribution function, and $\sigma$ is spin which corresponds to the energy eigenvalue $E_{k n}$. The real part $\varepsilon_{1}(\omega)$ can be calculated by the Kramers-Kronig 
transformation from its corresponding imaginary part as in the following ${ }^{6,31}$ :

$$
\varepsilon_{1}(\omega)=1+\frac{2}{\pi} \Phi \int_{0}^{\infty} \frac{\omega^{\prime} \varepsilon_{2}\left(\omega^{\prime}\right)}{\omega^{\prime 2}-\omega^{2}} \mathrm{~d} \omega^{\prime}
$$

where $\Phi$ is the integral principal value.

The absorption coefficient $\alpha(\omega)$ can be calculated $\mathrm{as}^{32}$

$$
\alpha^{i j}(\omega)=\frac{2 \omega k^{i j}(\omega)}{c},
$$

where $k^{i j}(\omega)$ is the extinction index, which can be expressed as the form ${ }^{32}$

$$
k^{i j}(\omega)=\frac{1}{\sqrt{2}}\left[\sqrt{\varepsilon_{1}^{i j}(\omega)^{2}+\varepsilon_{2}^{i j}(\omega)^{2}}-\varepsilon_{1}^{i j}(\omega)\right]^{1 / 2} .
$$

\section{RESULTS AND DISCUSSION}

The relaxed atomic structure of the monolayer GeS is illustrated in Fig. 1. We found that each unit cell of the monolayer GeS consists of two Ge atoms and two $\mathrm{S}$ atoms. Calculated results for the lattice parameters of the monolayer GeS are $a=3.675 \AA$, and $b=4.474 \AA$, which are consistent with previous theoretical calculations. ${ }^{2,7}$ In this work, to investigate the dependence of electronic and optical properties of monolayer $\mathrm{GeS}$ on the uniaxial strain, we apply the uniaxial strains individually along the zigzag $\varepsilon Z Z$ and armchair $\varepsilon A C$ directions. A uniaxial strain is applied by changing the lattice parameters $a$ and $b$ of the monolayer GeS at the equilibrium state. We define the strain via the lattice constants as $\varepsilon=\left(\tau-\tau_{0}\right) / \tau_{0}$, where $\tau$ and $\tau_{0}$, respectively, are the strained and equilibrium lattice constants of the monolayer GeS. In this paper, a uniaxial strain ranged from $-15 \%$ to $+15 \%$ was used in our calculations. The corresponding pressures at $15 \%$ of strain along the ZZ-strain and AC-strain are $8.46 \mathrm{GPa}$ and $4.03 \mathrm{GPa}$, respectively. Previous works indicated that the group-IV monochalcogenides, such as GeS and SnS, or black phosphorus

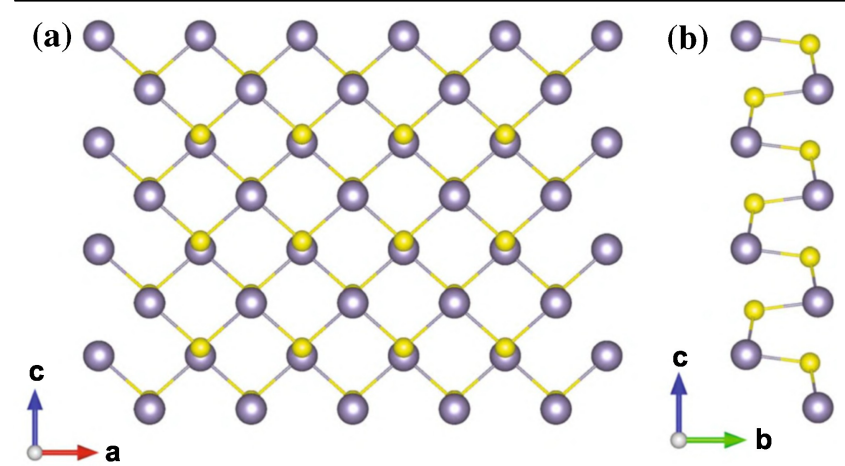

Fig. 1. The top view (a) and side view (b) of the atomic structure of the monolayer GeS. The gray and yellow balls stand for the $\mathrm{Ge}$ and $\mathrm{S}$ atoms, respectively. possess great mechanical flexibility and can sustain a large strain up to $27 \% .^{33,34}$ At the equilibrium state, our DFT calculations show that the total energy of monolayer $\mathrm{GeS}$ at equilibrium is $-135965.0 \mathrm{eV}$. Additionally, it should be noted that before investigating the effect of strain on the electronic and optical properties of the GeS monolayer, checking its stability is important. In order to evaluate the stability of the monolayer $\mathrm{GeS}$, we calculated its cohesive energy, which can be defined as follows: $E_{c o h}=\left(n_{G e} E_{G e}+n_{S} E_{S}-E_{t o t}\right) /\left(n_{G e}+n_{S}\right)$, where $n_{G e}$, and $n_{S}$ are, respectively, the number of Ge and $\mathrm{S}$ atoms, $E_{G e}, E_{S}$, and $E_{t o t}$ are the total energy of the Ge atom, $\mathrm{S}$ atom, and $\mathrm{GeS}$ monolayer, respectively. Our calculations indicated that the cohesive energy of the GeS monolayer at the equilibrium state is $-3.12 \mathrm{eV}$ per atom. This value is higher than the elementary substance of the $\mathrm{S}$ atom, $2.85 \mathrm{eV}$ per atom, resulting in the strong covalent bonding. Such structural stability results from the covalent $\sigma$ bond network between the chalcogenide atoms (S) and the metal atoms (Ge). Dependence of the total energy of the monolayer GeS on the uniaxial strains $\varepsilon Z Z / A C$ is shown in Fig. 2a. We can see that the effect of uniaxial strain on the total energy is negligible, for example, the total energies of $\mathrm{GeS}$ under AC-strain are $-135965.0 \mathrm{eV}$ and $-135963.9 \mathrm{eV}$ at the equilibrium state and under AC-strain of $\varepsilon A C=15 \%$, respectively. Besides, the difference in the total energy of the monolayer GeS in the presence of the $\varepsilon Z Z$ and $\varepsilon A C$ uniaxial strains is very small, especially in the case of tensile strain. Also, in order to improve the dynamically stability of the monolayer GeS, we also calculate its phonon dispersion curves at the equilibrium state, as shown in Fig. 2b. Our calculated results indicate that there are no soft phonon modes in the phonon spectrum of the monolayer GeS. It means that the monolayer GeS state is stable at equilibrium.

To consider deeply the effect of the uniaxial strains on the electronic properties of the monolayer GeS, we next calculate its electronic band structure under $\varepsilon Z Z$ and $\varepsilon A C$ uniaxial strain, as shown in Fig. 3. As shown in Fig. 3a, we can see that, at the equilibrium state, the monolayer $\mathrm{GeS}$ is an indirect band gap semiconductor with the energy band gap of $1.82 \mathrm{eV}$. This result is close to the previous PBE calculations $(1.73 \mathrm{eV})$ in Ref. 7. To understand the band formation in the GeS monolayer, we plot the total density of state (TDOS) and partial density of state (PDOS) of the monolayer GeS at the equilibrium state. We find that the valence bands near the Fermi level of the GeS monolayer are mainly contributed by the hybridization between the Ge-p, Ge-s, and S- $p$ orbitals. Whereas, the conduction bands near the Fermi level are mainly contributed by the Ge-p orbitals. Moreover, from Fig. 3a we can see that at the equilibrium state, the lowest unoccupied energy state (LUES) of the conduction bands is located on the $\Gamma-X$ path, whereas the highest 

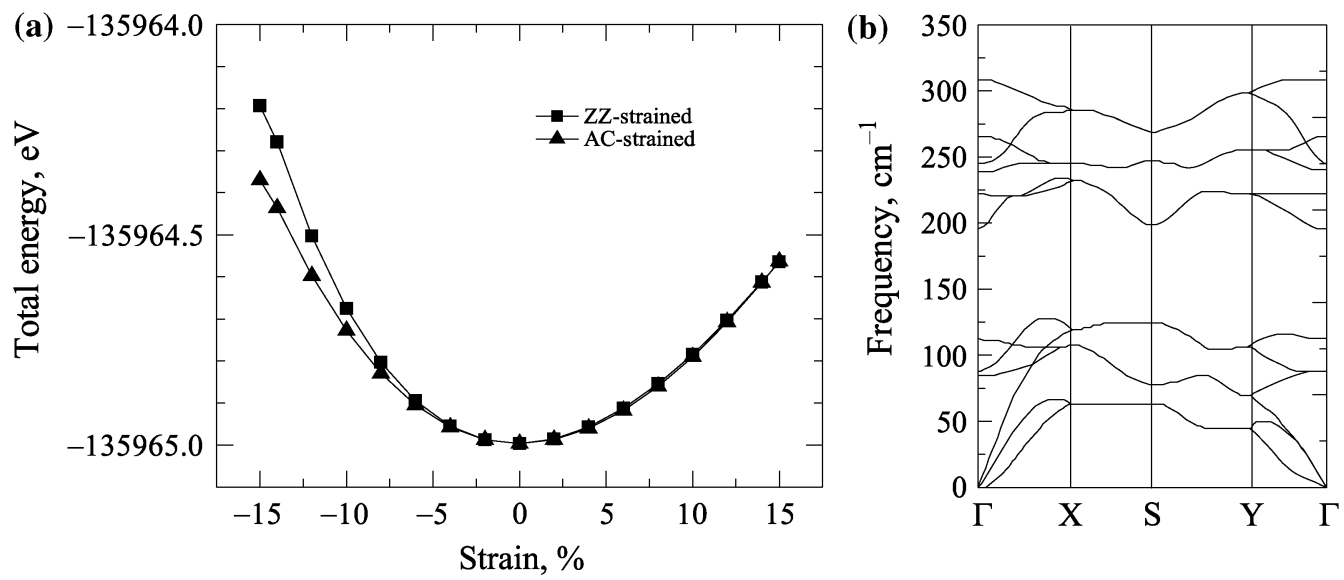

Fig. 2. Total energy of the monolayer GeS under uniaxial ZZ-strain $\varepsilon A C$ and uniaxial AC-strain $\varepsilon Z Z$ (a) and phonon dispersion spectra of monolayer GeS at equlibrium (b).
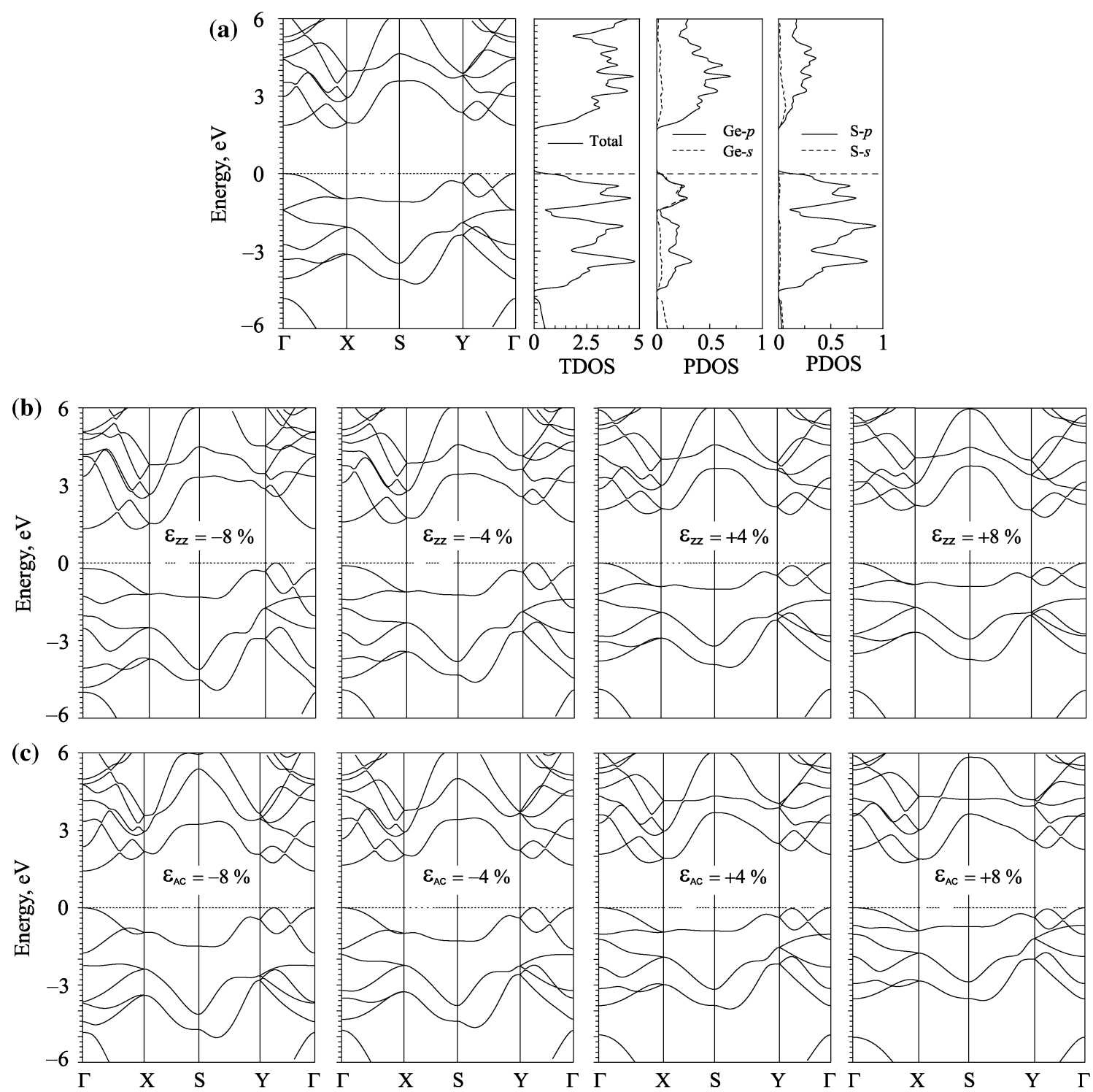

Fig. 3. (a) Electronic band structure, total density of states (TDOS), and partial density of states (PDOS) of the monolayer GeS at the equilibrium state. Electronic band structure of GeS under (b) $\varepsilon Z Z$ and (c) $\varepsilon A C$ uniaxial strain. 
occupied energy state (HOES) of the valence bands lies on the $Y-\Gamma$ path. However, the computed result for the band gap problem depends strongly on the computational approach. For example, while the calculated results of band gap using PBE functional as above-mentioned was $1.73 \mathrm{eV}$, the band gap of monolayer $\mathrm{GeS}$ at equilibrium calculated by HeydScuseria-Ernzerhof (HSE06) was up to $2.43 \mathrm{eV}^{7}$ or $2.74 \mathrm{eV}^{2}$ However, except for an energy shift, HSE06 and PBE calculations give very similar general features of electronic structure. ${ }^{7}$ It shows that the PBE method is good at predicting correct trends and physical mechanisms monolayer GeS. Therefore, in this study, we have only calculated the electronic properties of the monolayer GeS using the PBE functional. From Fig. $3 b$ and $c$ we can see that uniaxial strains modulate significantly the electronic band structure of the monolayer GeS, especially in the case of the compression strain. Interestingly, while the monolayer $\mathrm{GeS}$ is still a semiconductor with an indirect energy band gap under the ZZ-strain, the AC-strain $\varepsilon A C$ in monolayer GeS can lead to the appearance of an indirectdirect band gap transition as shown in Fig. 3c. The band structure and the density of states of the monolayer $\mathrm{GeS}$ in the presence of the strains $\varepsilon_{Z Z / A C}$ are shown in Figs. 3 and 4, respectively. The physical mechanism of the modulation in the energy gap of the monolayer GeS under uniaxial strains can be explained by analyzing in detail the position of the Fermi level. For example, it can be seen from Fig. $3 \mathrm{~b}$ that under the tensile strain the HOES of the valence bands is downshifted far from the Fermi level. Moreover, when the tensile strain $\varepsilon z z$ is smaller than $4 \%$, we can see that the conduction band minimum (CBM) of the monolayer $\mathrm{GeS}$ is shifted upward from the Fermi level, leading to an increase in its band gap. When the tensile $\varepsilon z z$ is larger than 4\%, the LUES of the conduction bands is changed from the $\Gamma-X$ path to the $Y-\Gamma$ path, resulting in a decrease in the energy gap of monolayer GeS. Besides, the ZZ-strain in the case of compression affects more on the valence band which is located at the $\Gamma$ point. The HOES of the valence bands of monolayer GeS is now changed from the $Y$ $\Gamma$ path to the $\Gamma$ point. The effect of the AC-strain on the band structure of the monolayer GeS is shown Fig. 3c. It can be firstly seen that the AC-strain $\varepsilon A C$ has a weak effect on the VBM of the monolayer GeS. In the case of $\varepsilon A C<0$ (compression), we found that the LUES of the conduction bands of the monolayer $\mathrm{GeS}$ is still now located at the $\Gamma$ point. By decreasing the compression strain in the case AC-strain $\varepsilon A C$, the LUES is shifted downward to the Fermi level, leading to a decrease in the energy gap in the monolayer GeS. However, the AC-strain in the case of tension has little effect on the LUES of the monolayer GeS. It indicates that in the case of $\varepsilon A C>0$, the band gap of the monolayer $\mathrm{GeS}$ is almost unchanged. In Fig. 5, we show the effect of the uniaxial strains on the energy band gap of the monolayer GeS. We can see that, under the compressive strain, the band gap of monolayer GeS depends linearly on the elongation in both directions of the uniaxial strains $\varepsilon Z Z$ and $\varepsilon A C$. In the case of tensile strain, while the band gap of the monolayer GeS depends strongly on uniaxial strain $\varepsilon Z Z$, the effect of AC-strain $\varepsilon A C$ on the band gap of the monolayer $\mathrm{GeS}$ is negligible. When $\varepsilon A C>0$, the band gap of monolayer GeS is around $1.80 \mathrm{eV}$. In the range of strain from $-15 \%$ to $15 \%$, the maximum band gap of monolayer $\mathrm{GeS}$ under uniaxial strain is $1.94 \mathrm{eV}$ at $\varepsilon Z Z=4 \%$.

To quickly consider optical properties of uniaxial strain monolayer $\mathrm{GeS}$, we calculate dielectric function and absorption coefficient of monolayer $\mathrm{GeS}$ under uniaxial strains in the energy range from $0 \mathrm{eV}$ to $8 \mathrm{eV}$. Effect of uniaxial strains on the dielectric constant of monolayer $\mathrm{GeS}$ is shown in Fig. 6. We can see that, at the equilibrium state, the dielectric constant of the monolayer $\mathrm{GeS}$ is 3.61. In the range from $0 \mathrm{eV}$ to $2 \mathrm{eV}$, the $\mathrm{AC}$-strain $\varepsilon A C$ affect strongly the dielectric constant of the monolayer $\mathrm{GeS}$ in comparison with the case of the ZZstrain $\varepsilon Z Z$. At energy of $0 \mathrm{eV}$, for example, under AC-strain from $\varepsilon A C=-8 \%$ to $\varepsilon A C=8 \%$, the dielectric constant of strained monolayer $\mathrm{GeS}$ varies from 3.17 to 4.17 , while the dielectric constant of $\mathrm{ZZ}$ strained monolayer GeS is only in the range from 3.54 to 3.73 for the same range of strain along the zigzag direction. From Fig. 6, we can see that the spectra have strong anisotropy in both real $\varepsilon_{1}(\omega)$ and imaginary $\varepsilon_{2}(\omega)$ parts of dielectric function of monolayer GeS. Besides, in comparison with the ZZstrained case, we can observe some new peaks in the imaginary part of the dielectric function of monolayer GeS under strain along the armchair direction as shown in the bottom panel of Fig. $6 \mathrm{~b}$. It means that we have new interband transitions in the monolayer GeS due to the presence of the AC-strain $\varepsilon A C$, especially in the case of compressive strain. These are transitions from the highest valence to the lowest conduction bands (see Fig. $3 b$ and c). Considering optical properties, especially absorption efficiency of materials, is very important for applications in solar energy conversion and optoelectronics. In Fig. 7, we calculate the absorption coefficient $\alpha(\omega)$ of monolayer GeS under uniaxial strains. As shown in Fig. 7a, we can see that the effect of ZZ-strain $\varepsilon Z Z$ on the absorption coefficient of monolayer GeS changes suddenly at an energy of $4.7 \mathrm{eV}$. Accordingly, the absorption coefficient is proportional to the elongation $\varepsilon Z Z$ in the energy region of less than $4.7 \mathrm{eV}$ and is inversely proportional to the elongation $\varepsilon Z Z$ in the energy region greater than $4.7 \mathrm{eV}$. In the range of energy greater than $6 \mathrm{eV}$, the absorption intensity tends to decrease. Furthermore, the effect of the strains on the absorption coefficient in this energy region (greater than $6 \mathrm{eV}$ ) is more apparent. 

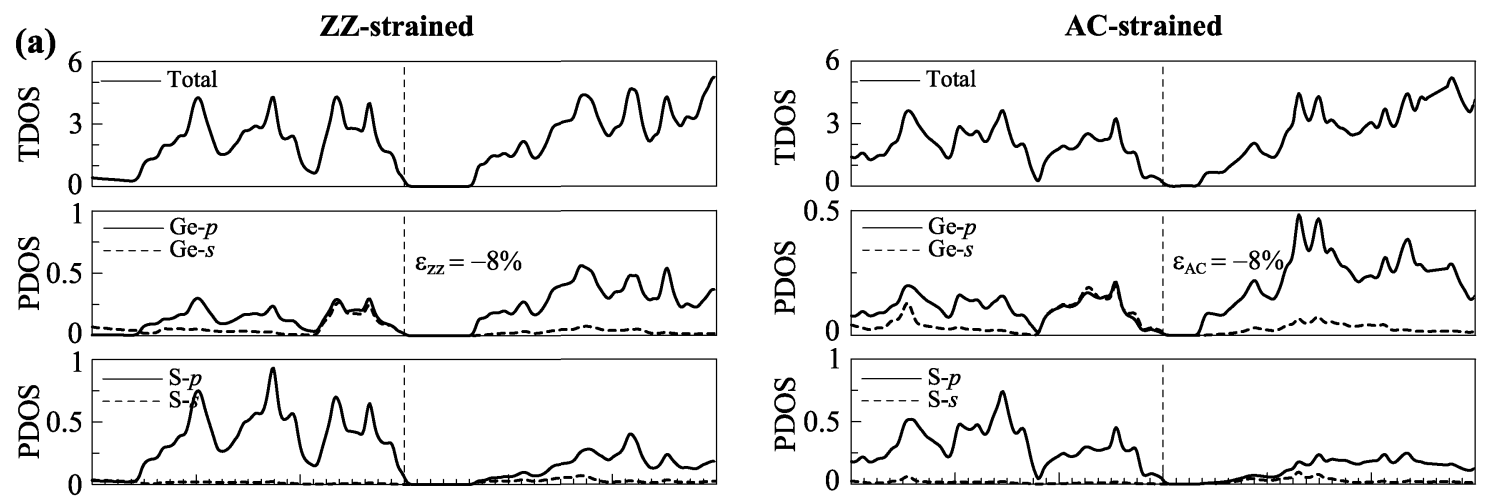

(b)
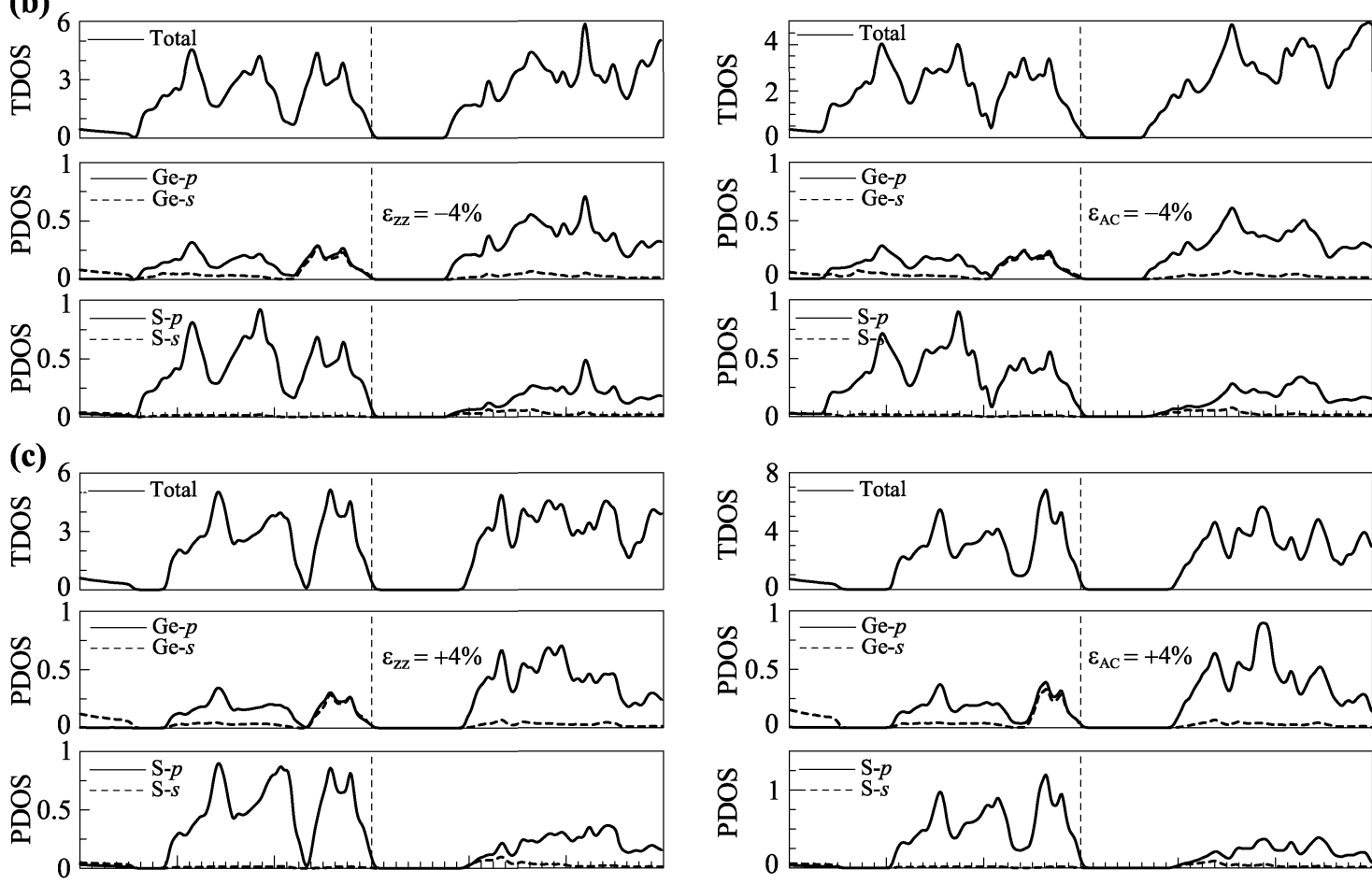

(d) 6
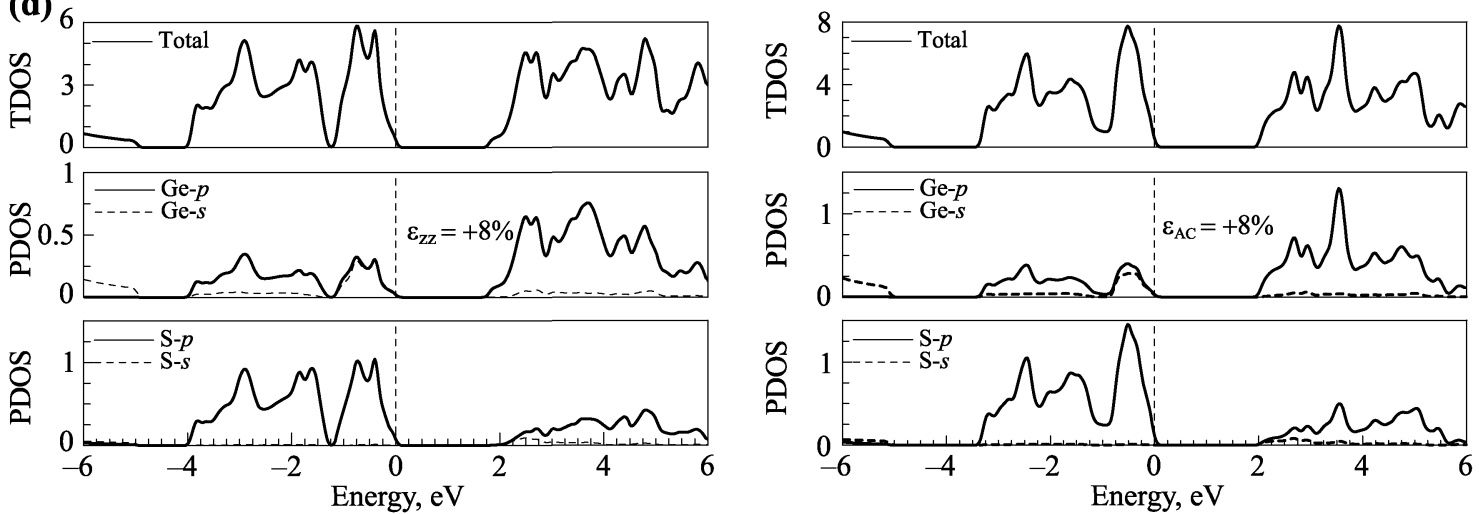

Fig. 4. Density of states (TDOS and PDOS) of the monolayer GeS under the ZZ-strain (left panel) and the AC-strain (right panel) at different elongations: (a) $\varepsilon=-8 \%$, (b) $\varepsilon=-4 \%$, (c) $\varepsilon=4 \%$, and (d) (a) $\varepsilon=8 \%$.

\section{CONCLUSION}

In conclusion, we have systematically studied the strain-tunable electronic and optical properties of monolayer GeS using first-principles calculations based on density functional theory. Our DFT calculations showed that the energy band gap of monolayer GeS depends strongly on the uniaxial strain in the case of compression strain, while the effect of 


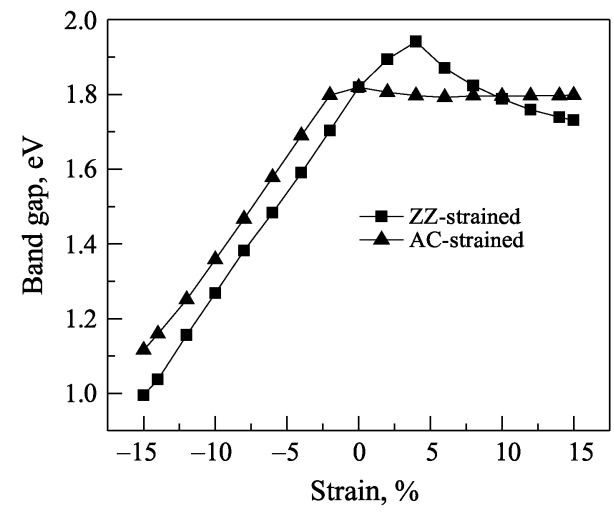

Fig. 5. Dependence of the band gap of the monolayer GeS on the uniaxial strain. the AC-strain in the case of tensile strain $\varepsilon A C>0$ is almost negligible. Also, the indirect-direct band gap transition was also observed in the monolayer GeS under the AC-strain $\varepsilon A C$. In the investigated energy regime from $0 \mathrm{eV}$ to $8.0 \mathrm{eV}$, the optical spectra of the monolayer $\mathrm{GeS}$ are highly anisotropic. The maximum absorption intensity is around $6 \mathrm{eV}$ in both directions of the uniaxial strains. Near this maximum point, the absorption coefficient is inversely proportional to the elongation $\varepsilon Z Z / A C$. According to these results, the monolayer GeS will exhibit extraordinary physical properties and present abundant opportunities for creating novel highperformance nanoelectronic and optoelectronic devices in the future.
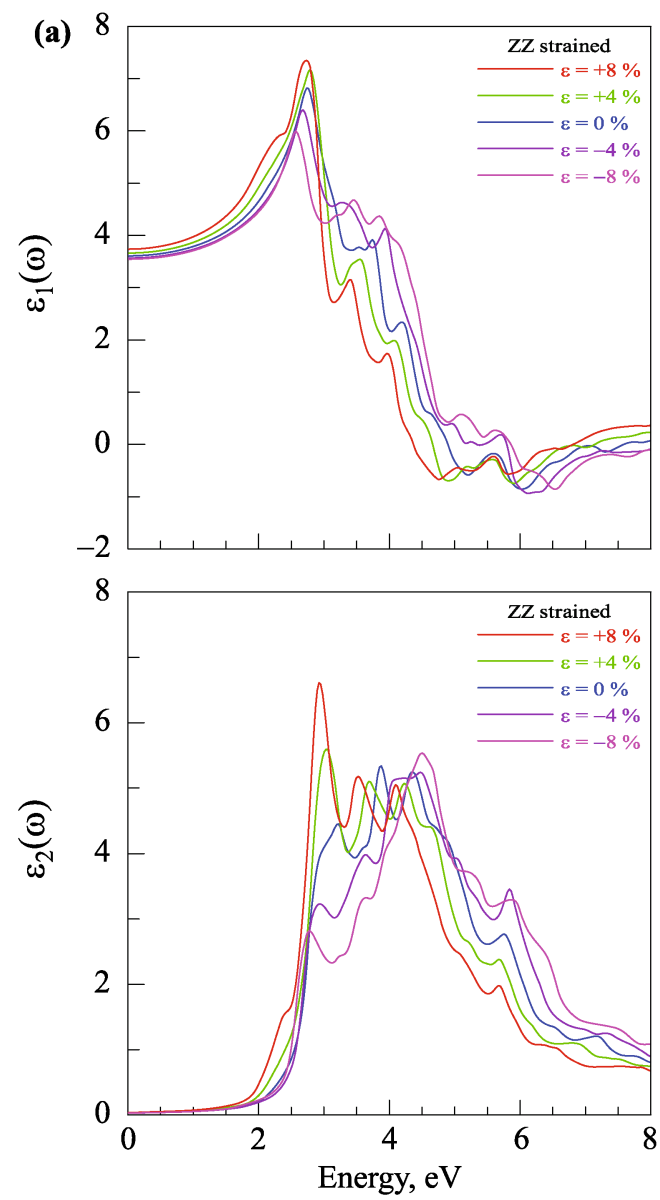
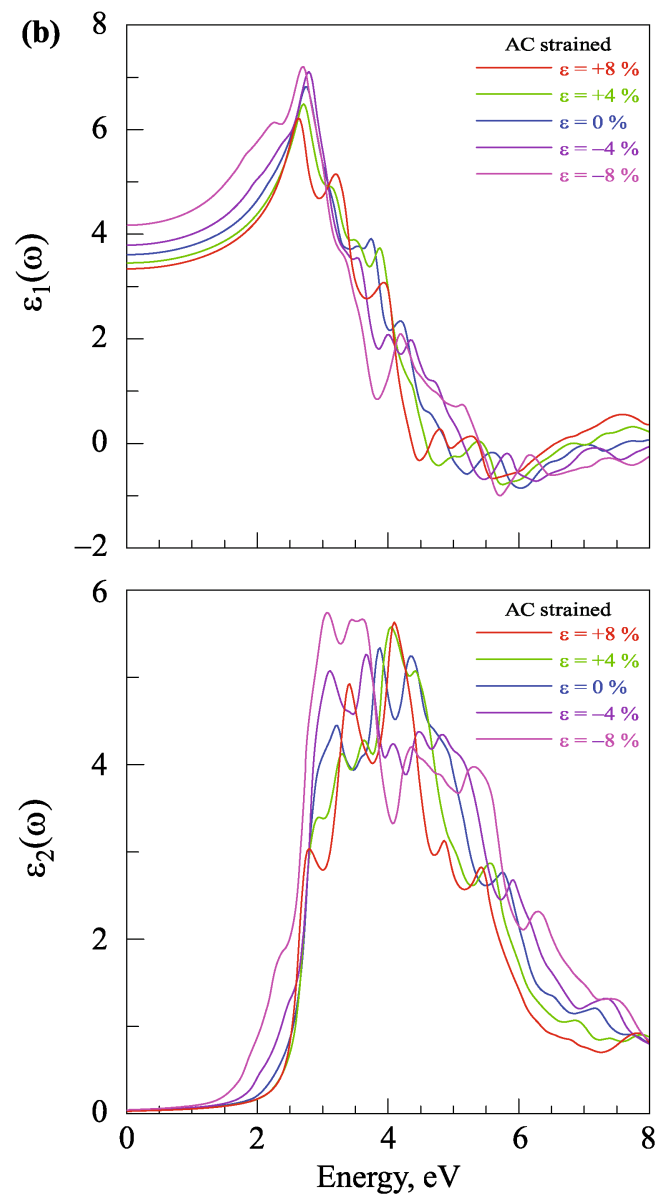

Fig. 6. Real $\varepsilon_{1}(\omega)$ and imaginary $\varepsilon_{2}(\omega)$ parts of dielectric function of the monolayer GeS at different elongations of the $Z Z$-strain $\varepsilon Z Z$ (a) and the AC-strain $\varepsilon A C(b)$. 

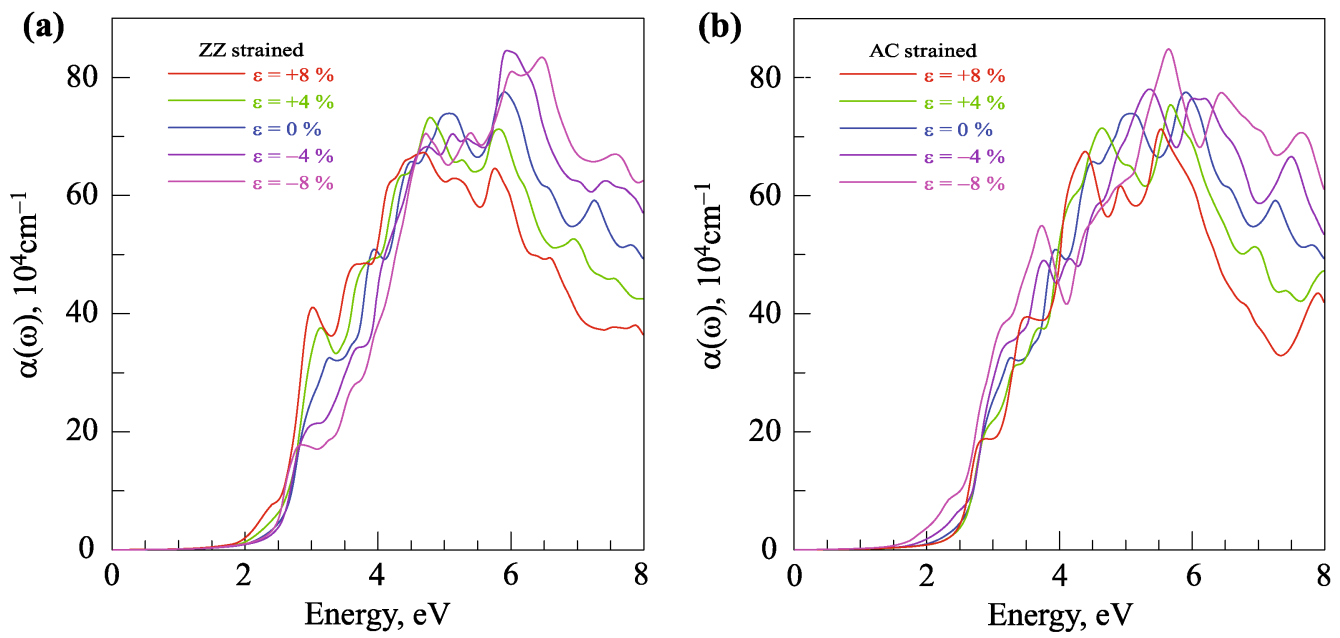

Fig. 7. Absorption coefficient $\alpha(\omega)$ of the monolayer GeS at different elongations of the ZZ-strain $\varepsilon Z Z$ (a) and the AC-strain $\varepsilon A C$ (b).

\section{ACKNOWLEDGMENTS}

This research is funded by the Vietnam National Foundation for Science and Technology Development (NAFOSTED) under Grant Number 103.012017.309 and the Belarusian Scientific Program "Convergence".

\section{CONFLICT OF INTEREST}

The authors declare that they have no conflict of interest.

\section{REFERENCES}

1. V. Sukhovatkin, S. Hinds, L. Brzozowski, and E.H. Sargent, Science 324(5934), 1542 (2009).

2. L. Xu, M. Yang, S.J. Wang, and Y.P. Feng, Phys. Rev. B 95, 235434 (2017).

3. L.D. Zhao, S.H. Lo, Y. Zhang, H. Sun, G. Tan, C. Uher, C. Wolverton, V.P. Dravid, and M.G. Kanatzidis, Nature 508, 373 (2014).

4. L.C. Gomes, A. Carvalho, and A.H. Castro Neto, Phys. Rev. $B$ 94, 054103 (2016).

5. S. Demirci, N. Avazli, E. Durgun, and S. Cahangirov, Phys. Rev. B 95, 115409 (2017).

6. S.Z. Karazhanov, P. Ravindran, A. Kjekshus, H. Fjellvag, and B.G. Svensson, Phys. Rev. B 75, 155104 (2007).

7. L. Huang, F. Wu, and J. Li, J. Chem. Phys. 144, 114708 (2016).

8. A.M. Cook, B.M. Fregoso, F. de Juan, S. Coh, and J.E. Moore, Nat. Commun. 8, 14176 (2017).

9. K.S. Novoselov, A.K. Geim, S.V. Morozov, D. Jiang, Y. Zhang, S.V. Dubonos, I.V. Grigorieva, and A.A. Firsov, Science 306, 666 (2004).

10. F. Li, X. Liu, Y. Wang, and Y. Li, J. Mater. Chem. C 4, 2155 (2016).

11. T. Yabumoto, J. Phys. Soc. Jpn. 13, 559 (1958),

12. A.K. Singh and R.G. Hennig, Appl. Phys. Lett. 105, 042103 (2014).

13. D.D. Vaughn, R.J. Patel, M.A. Hickner, and R.E. Schaak, J. Am. Chem. Soc. 132, 15170 (2010).

14. C. Li, L. Huang, G.P. Snigdha, Y. Yu, and L. Cao, ACS Nano 6,8868 (2012).

15. B. Mukherjee, Y. Cai, H.R. Tan, Y.P. Feng, E.S. Tok, and C.H. Sow, ACS Appl. Mater. Interfaces 5, 9594 (2013).

16. C. Chowdhury, S. Karmakar, and A. Datta, J. Phys. Chem. C 121, 7615 (2017).
17. H. Wang and X. Qian, 2D Mater. 4, 015042 (2017).

18. L.C. Gomes, A. Carvalho, and A.H. Castro Neto, Phys. Rev. $B$ 92, 214103 (2015).

19. L. Makinistian and E.A. Albanesi, Phys. Rev. B 74, 045206 (2006).

20. S. Zhang, N. Wang, S. Liu, S. Huang, W. Zhou, B. Cai, M. Xie, Q. Yang, X. Chen, and H. Zeng, Nanotechnology 27, 274001 (2016).

21. G. Guo and G. Bi, Micro Nano Lett. 13, 600 (2018).

22. K.D. Pham, C.V. Nguyen, H.V. Phuc, T.V. Vu, N.V. Hieu, B.D. Hoi, L.C. Nhan, V.Q. Nha, and N.N. Hieu, Superlattices Microstruct. 120, 501 (2018).

23. D. Tan, H.E. Lim, F. Wang, N.B. Mohamed, S. Mouri, W. Zhang, Y. Miyauchi, M. Ohfuchi, and K. Matsuda, Nano Res. 10, 546 (2017).

24. P. Giannozzi, S. Baroni, N. Bonini, M. Calandra, R. Car, C. Cavazzoni, D. Ceresoli, G.L. Chiarotti, M. Cococcioni, I. Dabo, A.D. Corso, S. de Gironcoli, S. Fabris, G. Fratesi, R. Gebauer, U. Gerstmann, C. Gougoussis, A. Kokalj, M. Lazzeri, L. Martin-Samos, N. Marzari, F. Mauri, R. Mazzarello, S. Paolini, A. Pasquarello, L. Paulatto, C. Sbraccia, S. Scandolo, G. Sclauzero, A.P. Seitsonen, A. Smogunov, P. Umari, and R.M. Wentzcovitch, J. Phys.: Condens. Matter 21, 395502 (2009).

25. J.P. Perdew, K. Burke, and M. Ernzerhof, Phys. Rev. Lett. 77(18), 3865 (1996).

26. J.P. Perdew, K. Burke, and M. Ernzerhof, Phys. Rev. Lett. 78,1396 (1997).

27. V.V. Ilyasov, C.V. Nguyen, I.V. Ershov, and N.N. Hieu, J. Appl. Phys. 117, 174309 (2015).

28. C.V. Nguyen, N.N. Hieu, and V.V. Ilyasov, J. Electron. Mater. 45, 4038 (2016).

29. H.V. Phuc, N.N. Hieu, B.D. Hoi, L.T.T. Phuong, N.V. Hieu, and C.V. Nguyen, Superlattices Microstruct. 112, 554 (2017).

30. H.V. Phuc, N.N. Hieu, B.D. Hoi, L.T.T. Phuong, and C.V. Nguyen, Surf. Sci. 668, 23 (2018).

31. A. Delin, P. Ravindran, O. Eriksson, and J. Wills, Int. J. Quantum Chem. 69, 349 (1998).

32. P. Ravindran, A. Delin, B. Johansson, O. Eriksson, and J.M. Wills, Phys. Rev. B 59, 1776 (1999).

33. M. Elahi, K. Khaliji, S.M. Tabatabaei, M. Pourfath, and R. Asgari, Phys. Rev. B 91, 115412 (2015).

34. L.C. Gomes, A. Carvalho, and A.C. Neto, Phys. Rev. B 92, 214103 (2015).

Publisher's Note Springer Nature remains neutral withregard to jurisdictional claims in published maps and institutionalaffiliations. 